\title{
¿Incide la forma de tenencia de la vivienda habitual sobre la situación de empleo en España?
}

\author{
JOSÉ E. RODRÍGUEZ HERNÁNDEZ \\ Departamento de Economía Aplicada, UNIVERSIDAD DE LA LAGUNA, ESPAÑA. E-mail: \\ jerodri@ull.es
}

JAVIER A. BARRIOS GARCÍA

Departamento de Economía Aplicada, UNIVERSIDAD DE LA LAGUNA, ESPAÑA. E-mail: jabarrio@ull.es

\begin{abstract}
RESUMEN
El objetivo de este trabajo es aportar mayor evidencia empírica sobre la posible existencia en España de relaciones causales entre el régimen de tenencia de la vivienda habitual (propiedad/alquiler) y la situación en el empleo del cabeza de familia (empleado/desempleado), deslindando a su vez este efecto del generado por otros covariantes e inobservables que puedan afectar, así como del efecto causal inverso que pueda existir. Para ello, basándonos en los microdatos anuales de la Encuesta de Condiciones de Vida para el año 2010 y bajo el enfoque de la literatura de efectos de un tratamiento, estimamos modelos switching probit que relacionan ambas variables a nivel microeconómico. Los resultados obtenidos nos permiten afirmar que poseer la vivienda habitual en propiedad, tanto si se tiene con hipoteca o sin ella, aumenta la probabilidad de empleo en torno a un $10 \%$.
\end{abstract}

Palabras clave: Forma de tenencia de la vivienda, desempleo, switching probit.

\section{Does the Housing Tenure Choice Affect the Employment Situation in Spain?}

\begin{abstract}
The objective of this study is to provide more empirical evidence on the possible existence in Spain of causal relationships between housing tenure choice (ownership/rental) and the employment status of household head (employed/unemployed), separating this effect from the one generated by other covariates and unobservables which might affect, as well as from the reverse causal effect that might exist. With this aim, based on micro data from the Survey of Living Conditions for 2010 and the approach of the literature of treatment effects, we estimate switching probit models that relate these variables at the microeconomic level. The results obtained allow us to assert that owning the main residence, whether it is mortgaged or not, increases the probability of employment around $10 \%$.
\end{abstract}

Keywords: Housing Tenure Choice, Unemployment, Switching Probit.

Clasificación JEL: C35, R20

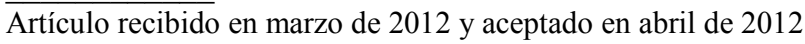

Artículo disponible en versión electrónica en la página www.revista-eea.net, ref. ə-30201 


\section{INTRODUCCIÓN}

En el contexto español actual de crisis económica con alto nivel de desempleo $(20,07 \%$ en $2010,21,6 \%$ en 2011 , promedios trimestrales. Fuente: E.P.A. I.N.E.), organismos europeos animan a España a estimular la movilidad laboral y la flexibilidad salarial como factores claves para poder revertir esta situación. Al mismo tiempo, dados los estrictos compromisos de reducción del déficit público contraídos por el gobierno de la nación en el marco de la UE, cualquier medida de política económica que persiga favorecer la reducción del desempleo debe ser escrutada profundamente antes de ser puesta en funcionamiento, sopesando cuidadosamente los beneficios y costes de su implementación, tanto a nivel de presupuesto público como de empleo. En este sentido, con objeto de favorecer la movilidad geográfica de los trabajadores, el gobierno actual se plantea la posibilidad de intervenir sobre el mercado de vivienda mediante el establecimiento de una posible deducción por alquiler de vivienda. El objetivo del presente trabajo es poner de manifiesto si este tipo de medidas están justificadas en términos de fomento del empleo, dejando a un lado los aspectos presupuestarios o redistributivos de este tipo de disposiciones.

En la literatura, han sido múltiples los trabajos que han evidenciado diversos vínculos entre el mercado de vivienda y el mercado laboral. Bover et al. (1989) señalan al menos cinco fuentes de influencia del mercado de vivienda sobre el mercado de trabajo: el efecto de la elección de tenencia de la vivienda sobre la movilidad laboral, el efecto del coste de vida sobre los individuos, el efecto de coste de localización para las empresas, el efecto riqueza sobre el gasto regional $\mathrm{y}$, finalmente, el efecto sobre las ganancias futuras esperadas derivadas de los movimientos en los precios del suelo y de la vivienda. Ioannides y Loury (2004) también destacan el "efecto vecindario" como otra fuente de influencia del mercado de vivienda sobre el de trabajo, que se deriva de las redes de información de empleo que se generan en el vecindario y que pueden favorecer la búsqueda de empleo. A estos efectos cabría añadir el aspecto incentivador que puede tener el ser propietario de la vivienda habitual con una hipoteca pendiente sobre la búsqueda de empleo (Flatau et al., 2003, y Rouwendal y Nijkamp, 2010), así como la conexión del sector de la construcción con el mercado laboral, la cual ha jugado específicamente un papel de primera magnitud en la reciente evolución del desempleo en España (García, 2012). En sentido contrario, el mercado laboral influye también en el de vivienda fundamentalmente a través del efecto que posee la renta permanente o a largo plazo sobre las decisiones de los individuos en materia de vivienda.

De entre las distintas influencias apuntadas del mercado de la vivienda en el mercado de trabajo, la que ha sido objeto de un mayor análisis es el efecto restrictivo que la tenencia de vivienda en propiedad genera sobre la movilidad laboral, como consecuencia de los mayores costes de desplazamiento que so- 
portan los propietarios de vivienda ${ }^{1}$. No obstante, aunque la existencia de mayores costes de desplazamiento puede lastrar teóricamente la situación de empleo de los propietarios de vivienda debido a su mayor fijación geográfica, este efecto debe confrontarse con el resto de fuerzas intervinientes, especialmente con la existencia de posibles efectos de vecindario o incluso financieros a través de la hipoteca, que puedan actuar en dirección contraria. En este sentido, el efecto final de la forma de tenencia de la vivienda habitual sobre la situación de empleo del cabeza de familia debe ser por lo tanto una cuestión empírica.

Es precisamente este aspecto el que pretendemos abordar en el presente trabajo. Para ello, utilizando los microdatos de la Encuesta de Condiciones de Vida (ECV) para el año 2010 y bajo el enfoque de la literatura de efectos de un tratamiento, nuestro objetivo será contrastar estas argumentaciones teóricas aportando evidencia empírica a nivel microeconómico sobre la posible existencia en España de relaciones causales entre la forma de tenencia de la vivienda habitual (propiedad/alquiler) y la situación en el empleo del cabeza de familia (empleado/desempleado) ), deslindando a su vez este efecto del generado por otros covariantes e inobservables que puedan afectar, así como del efecto causal inverso que pueda existir. La ausencia de estudios microeconómicos que aborden la implicación de la tenencia de vivienda sobre el mercado laboral en España, convierte a este trabajo, junto con Barceló (2006) en los únicos antecedentes conocidos, si bien en Barrios y Rodríguez (2004) se analiza el mismo problema a nivel agregado español con datos provinciales.

El trabajo se organiza en cinco apartados. En el apartado 2 destacamos los principales antecedentes. El apartado 3 describe los datos y variables utilizadas, así como la estrategia empírica adoptada y el modelo econométrico empleado. En el siguiente apartado mostramos los resultados obtenidos de la estimación de los modelos. Finalmente, en el último apartado destacamos las principales conclusiones obtenidas, recogiendo en un apéndice final los estadísticos descriptivos y la definición de las variables empleadas.

\section{ANTECEDENTES}

Las evidencias empíricas obtenidas a nivel microeconómico sobre los posibles vínculos entre el mercado de vivienda y el mercado de trabajo resultan contrapuestas, $\mathrm{y}$ se han fundamentado principalmente en tres modelos teóricos diferenciados, según el punto de vista con que se aborde el mercado laboral.

En primer lugar podemos citar los modelos Walrasianos como el de Oswald (1997), donde se argumenta que la tenencia en propiedad de la vivienda genera

\footnotetext{
${ }^{1}$ Véase Van Ewijk y Van Leuvenjstein (2009) donde se recopilan una serie de estudios realizados a nivel europeo sobre el efecto que la tenencia de vivienda genera sobre la movilidad laboral y la duración en el desempleo.
} 
un efecto negativo sobre el empleo, al limitar la movilidad de los trabajadores debido a los mayores costes de desplazamiento que soportan los propietarios frente a los inquilinos. Esta es la que se conoce comúnmente hoy en día como "tesis de Oswald" (Oswald, 1996). Por otra parte, se encuentran los modelos de equilibrio general o estructuralistas (Layard et al., 1991, Phelps, 1994), que refuerzan la tesis anterior al considerar la movilidad geográfica un factor estructural que influye sobre los salarios y precios (Nickell, 1998). El tercer marco teórico lo constituye los modelos deudores de la teoría de búsqueda de trabajo (Pissarides, 2000, Coulson y Fisher, 2002, 2009, Dohmen, 2005, Munch et al., 2006, 2008 o Rouwendal y Nijkamp, 2010), basados en la naturaleza no perfectamente competitiva del mercado laboral.

Bajo estos tres enfoques teóricos, una de las cuestiones más analizadas en la literatura ha sido el efecto restrictivo que la tenencia de vivienda en propiedad genera sobre la movilidad laboral. Cabe citar, entre otros, los trabajos desarrollados para el Reino Unido de Böheim y Taylor (2002) donde se evidencia que los desempleados y los inquilinos presentan mayor propensión a desplazarse, Hughes y McCormick (1987), que ponen de manifiesto que las viviendas públicas limitan la movilidad residencial, y Henley (1998) quien concluye que las caídas en el valor de la vivienda limitan la capacidad de desplazamiento de los propietarios a la hora de buscar una nuevo empleo.

Por otro lado, los trabajos de Brunet y Lesueur (2009) y Green y Hendershott (2003) para Francia y Estados Unidos, respectivamente, confirman la tesis de Oswald. En cambio, los resultados obtenidos por Van Leuvensteijn y Koning (2004) para los países bajos y Coulson y Fisher (2002) para Estados Unidos rechazan el argumento de Oswald, al igual que Flatau et al. (2003) para Australia y Rouwendal y Nijkamp (2010) para los Países Bajos. En estos dos últimos trabajos igualmente se evidencia que los propietarios que poseen una hipoteca presentan menor probabilidad de estar desempleados y un menor periodo de paro.

En el caso español, cabe destacar como únicos antecedentes, el trabajo que realiza a nivel agregado Barrios y Rodríguez (2004) con datos del año 1991 en el que se concluye que las provincias españolas con una tasa de viviendas en propiedad 10 puntos porcentuales mayor poseen tasas de desempleo menores en 2.2 puntos porcentuales, aproximadamente, así como la contribución de Barceló (2006) ahora con datos micro, y en las que empleando modelos de duración concluye que los propietarios y los inquilinos de viviendas de protección oficial son los que presentan una menor movilidad laboral.

Respecto a estos antecedentes, este trabajo realiza importantes aportaciones. En primer lugar, el estudio de la incidencia del mercado de vivienda sobre el mercado de trabajo se aborda considerando explícitamente la endogeneidad de la tenencia de vivienda, a diferencia de las contribuciones de Coulson y Fisher 
(2002), (2009) o Flatau et al. (2003), entre otros. En segundo lugar, analiza la relación entre ambos mercados a nivel microeconómico bajo una estrategia empírica basada en el enfoque de la literatura de efectos de un tratamiento mediante la estimación de modelos switching probit y empleándose microdatos recientes (año 2010). En tercer lugar, se analiza en profundidad el papel que desempeña la tenencia de hipoteca y la antigüedad en la vivienda como posibles moduladores de la influencia del mercado de vivienda sobre el mercado de trabajo.

\section{DATOS, VARIABLES Y MODELO ECONOMÉTRICO}

\subsection{Datos}

En este trabajo hemos empleado los microdatos anuales a nivel nacional contenidos en la Encuesta de Condiciones de Vida (E.C.V. Fuente: INE) para el año 2010, considerando en la muestra final sólo aquellos hogares donde la persona responsable de la vivienda posee entre 18 y 65 años de edad, y es económicamente activa, excluyendo los que habitan en una vivienda con alquiler inferior al de mercado ${ }^{2}$ o en cesión gratuita, así como aquellos hogares con observaciones perdidas en cualquiera de las variables consideradas. De igual forma, se han eliminado aquellos hogares que se han mudado a su vivienda actual durante el año en que son encuestados o el año inmediatamente anterior, para evitar el problema de la causalidad inversa (esto es, que las variables relacionadas con los resultados laborales de la persona responsable de la vivienda influyan simultáneamente sobre la forma de tenencia de la vivienda). En la Tabla 1 recogemos los tamaños muestrales empleados, según el tipo de tenencia y la situación laboral del cabeza de familia.

Tabla 1

Tamaño muestral (Total observaciones)

\begin{tabular}{lcccc}
\hline & & Desempleados & Empleados & Total \\
\hline Inquilinos & $\mathbf{2 0 2}$ & $\mathbf{5 3 9}$ & $\mathbf{7 4 1}$ \\
Propietarios & $\mathbf{8 1 1}$ & $\mathbf{4 9 9 4}$ & $\mathbf{5 8 0 5}$ \\
& sin hipoteca & 427 & 2256 & 2683 \\
& con hipoteca & 384 & 2738 & 3122 \\
Total & & $\mathbf{1 0 1 3}$ & $\mathbf{5 5 3 3}$ & $\mathbf{6 5 4 6}$ \\
\hline
\end{tabular}

Fuente: Elaboración propia a partir de los datos procedentes de la Encuesta de Condiciones de Vida para el año 2010 (I.N.E.)

\footnotetext{
${ }^{2}$ Se ha excluido de la muestra analizada los hogares con alquileres a precio inferior al de mercado, entre los que se incluyen las viviendas de renta antigua y el alquiler público, al tratarse de hogares que por sus características presentan una menor movilidad geográfica, lo que podría distorsionar las conclusiones del trabajo.
} 


\subsection{Variables empleadas}

La situación laboral del cabeza de familia y el régimen de tenencia de la vivienda habitual se recoge a través de las variables binarias EMPLEO y TENEN respectivamente. La variable EMPLEO adopta el valor 1 si el individuo se encuentra empleado en el instante de realizar la encuesta, siendo 0 en otro caso, mientras que el valor 1 en la variable TENEN indica propiedad y 0 alquiler.

Por otro lado, para la ecuación de tenencia hemos considerado variables explicativas similares a las utilizadas en Barrios y Rodríguez (2005) o Rodríguez y Barrios (2007) donde incluyen como principales determinantes variables socioeconómicas como: la edad del cabeza de familia, su sexo, estado civil y nivel de estudios, número de miembros del hogar, índices de precios hedónicos relativos de vivienda en propiedad y en alquiler por comunidades autónomas ${ }^{3}$, así como indicadores de la comunidad autónoma de residencia en función del nivel de precios de la vivienda. Adicionalmente, hemos incorporado variables indicadoras de nacimiento en España, de antigüedad en la vivienda actual, del número de años anteriores en trabajo remunerado, $\mathrm{y}$ un indicador que clasifica la comunidad autónoma de residencia según la tasa de paro. Aunque en los trabajos citados anteriormente queda patente que la renta permanente o a largo plazo de los hogares es una variable determinante en la elección de forma de tenencia de la vivienda, en este trabajo decidimos excluirla como variable explicativa debido fundamentalmente a los problemas de endogeneidad potencial que podría acarrear con la variable dependiente de empleo/desempleo analizada, y teniendo presente que nuestro primer objetivo es el estudio de esta última variable y no específicamente el modelizar la elección de tenencia de vivienda, suficientemente tratada en la literatura ${ }^{4}$.

Para seleccionar las variables explicativas de la ecuación de empleo hemos tomado como referencia los trabajos de Bentolila y Blanchard (1990), Layard et

${ }^{3}$ Los índices de precios hedónicos por CC.AA. se elaboran como en Barrios y Rodríguez (2005) o Rodríguez y Barrios (2007). Para ello se han empleado los datos de la Encuesta de Presupuestos Familiares (base 2006), Fuente: I.N.E., debido a que esta fuente incluye un mayor detalle de las características de la viviendas que la ECV aquí utilizada. En las estimaciones realizadas se ha utilizado el índice calculado para el año inmediatamente anterior (2009). Los resultados pueden ser facilitados bajo petición.

${ }^{4}$ Cabe señalar que si estimamos los mismos modelos incluidos en la Tabla 2 añadiendo como variable explicativa en la ecuación de tenencia de la vivienda una variable indicadora de la capacidad económica del hogar como la renta corriente del mismo correspondiente al año 2009 (en logaritmo), los parámetros de efectos de tratamiento medios estimados (Tabla 3) se incrementan, por ejemplo para la muestra de propietarios vs alquiler de mercado hasta unos valores de $\Delta^{\mathrm{ATE}}=0.165, \Delta^{\mathrm{ATT}}=0.157, \Delta^{\mathrm{ATU}}=0.22$, todos ellos significativos al $1 \%$ de probabilidad, reforzándose las conclusiones del presente trabajo. No obstante, este resultado es cuestionable puesto que se comprueba que no se puede rechazar que esta variable de renta sea exógena al $1 \%$ de significatividad mediante el test de endogeneidad propuesto por Rivers y Vuong (1988). 
al (1991), Blanchflower y Oswald (1994), o Coulson y Fisher (2002), (2009), entre otros, incluyéndose: edad, sexo y educación del cabeza de familia, su localización en una comunidad autónoma según la tasa de paro regional, así como el número de años pasados con anterioridad en trabajo remunerado del cabeza de familia ${ }^{5}$.

La definición de las variables y los correspondientes estadísticos descriptivos se muestran en el apéndice final (Tabla A1 y Tabla A2).

\subsection{Estrategia empírica y modelo econométrico}

El objetivo principal de este trabajo es contrastar empíricamente la posible existencia del efecto que pueda tener la forma de tenencia de la vivienda habitual sobre la situación de empleo/desempleo del cabeza de familia, deslindándolo del efecto causal inverso que pueda existir. Para ello adoptamos la siguiente estrategia empírica:

a) Para evitar el problema de la causalidad inversa, como se indica en el apartado 3.1, se incluyen en la muestra solo aquellos hogares que han permanecido en la vivienda actual al menos durante los dos últimos años 2009 y 2010. De esta manera, entendemos que se garantiza que la situación actual de empleo no ha influido en la decisión de tenencia de la vivienda actual. A esto debemos añadir que la selección del período muestral año 2010, debe ayudar a la estimación del efecto, si existiera, debido a que el intenso proceso de destrucción de empleo habido en España desde 2008 promueve la existencia de variabilidad en la variable resultado analizada (empleo/desempleo), manteniéndose la decisión de tenencia de vivienda anterior a esta situación.

b) Teniendo en cuenta que el resultado analizado es binario (empleo/desempleo) y que debido a la naturaleza de la Encuesta de Condiciones de Vida pueden existir factores inobservados comunes relevantes que influyan a la vez tanto en la decisión de tenencia de la vivienda como en la situación de empleo/desempleo (problema de endogeneidad de la tenencia de vivienda), optamos por utilizar el modelo probit bivariante (véase Wooldridge, 2010, p. 594-599) o el más general switching probit que tiene a este como un caso particular, y que no se había utilizado antes en la literatura especializada en este tema.

c) Por último, enmarcamos el estudio dentro del enfoque de la literatura sobre efectos de un tratamiento, precisamente porque el objetivo es intentar deslindar el efecto individual que la forma de tenencia de la vivienda habitual (alquiler vs propiedad) genera sobre la situación de empleo del cabeza de familia económicamente activo (trabajando/desempleado) del

${ }^{5}$ Las variables excluidas por no resultar estadísticamente significativas han sido testeadas mediante un test de razón de verosimilitud entre el modelo estimado con y sin dichas variables. 
efecto de otros covariantes que puedan influir (edad, estudios, etc.), así como de los factores inobservados comunes que puedan afectar. Esto es, perseguimos analizar como sería la probabilidad de empleo de un mismo individuo con unas determinadas características (observadas y no observadas) tanto si posee la vivienda habitual en propiedad como si dispone de ella en alquiler. Esto es lo que podríamos denominar como generación del contrafactual porque sólo se observa uno de los dos estados (alquiler o propiedad) para cada individuo. Sintetizaremos estos efectos de tratamiento individuales con los habituales parámetros de efectos medios o distribucionales (Heckman et al., 1999, Heckman et al., 2003, Aakvik et al., 2005).

En consecuencia, para desarrollar la estrategia empírica propuesta, supondremos una muestra de individuos iid extraída aleatoriamente de una población, donde $Y_{\mathrm{o} i}$ e $Y_{1 i}$ son los valores que adopta la situación de empleo/desempleo del cabeza de familia si el hogar $i$ reside en su vivienda habitual en alquiler o en propiedad, respectivamente. Desde la óptica contrafactual, supondremos que $Y_{\mathrm{o} i}$ e $Y_{1 i}$ están definidos para todos los individuos y que estos resultados son independientes entre ellos de tal forma que no existe interacción entre los agentes ${ }^{6}$.

La forma de tenencia de la vivienda habitual viene expresada por la variable binaria $T_{i}\left(T_{i}=1\right.$ si lo es en propiedad, $T_{i}=0$ si lo es en alquiler), de modo que la elección entre ambas opciones se genera mediante un modelo probit, que en su versión de variable latente está dado por (1), con $Z_{i}$ un vector de características del individuo o de las alternativas de tenencia observadas, $\beta_{T}$ un vector de parámetros conforme, y $u_{T i}$ un término de error aleatorio que refleja el impacto de factores no observados sobre la elección de tenencia de vivienda que se supone normal con media cero e independiente de $Z$.

$$
T_{i}=1\left(T_{i}^{*}=\beta_{T}^{\prime} Z_{i}+u_{T i} \geq 0\right)= \begin{cases}1, & \text { si } T_{i}^{*}>0 \\ 0, & \text { si } T_{i}^{*} \leq 0\end{cases}
$$

De igual forma, consideramos el resultado laboral (empleo/desempleo) como una variable binaria, que se relaciona con las variables observadas y no observadas para propietarios e inquilinos mediante respectivos modelos probit de la forma (2) y (3), verificándose que los términos de error en (1), (2) y (3) son normales trivariantes con sus varianzas normalizadas a la unidad según (4), con lo cual estaremos ante el modelo comúnmente denominado como switching probit (O’Higgins, 1994, Carrasco, 2001, Aakvik et al., 2005).

\footnotetext{
${ }^{6}$ Se asume la no existencia de efectos de equilibrio general. Heckman, et al. (1998) muestran los problemas que genera ignorar estas interacciones a gran escala.
} 


$$
\begin{gathered}
Y_{0 i}=1\left(Y_{0 i}^{*}=\beta_{0}^{\prime} X_{i}+u_{0 i} \geq 0\right)= \begin{cases}1, & \text { si } Y_{0 i}^{*}>0 \\
0, & \text { si } Y_{0 i}^{*} \leq 0\end{cases} \\
Y_{1 i}=1\left(Y_{1 i}^{*}=\beta_{1}^{\prime} X_{i}+u_{1 i} \geq 0\right)= \begin{cases}1 & , \text { si } Y_{1 i}^{*}>0 \\
0 & , \text { si } Y_{1 i}^{*} \leq 0\end{cases} \\
\left(\begin{array}{l}
u_{T} \\
u_{0} \\
u_{1}
\end{array}\right) \sim \mathrm{N}\left(0, \Sigma=\left(\begin{array}{ccc}
1 & \rho_{T 0} & \rho_{T 1} \\
\rho_{T 0} & 1 & \rho_{01} \\
\rho_{T 1} & \rho_{01} & 1
\end{array}\right)\right)
\end{gathered}
$$

El modelo probit bivariante (véase por ejemplo Wooldridge, 2010, p. 595), para el que el resultado laboral de propietarios e inquilinos coincide, constituirá un caso particular del modelo switching probit (1), (2) y (3) con $\beta_{0}=\beta_{1} \mathrm{y}$ $\rho_{T 0}=\rho_{T 1}$, pudiéndose testear frente a este por tanto mediante un test de ratio de verosimilitudes.

La estimación del modelo switching probit se realiza maximizando la correspondiente función de verosimilitud:

$$
\begin{gathered}
\log L= \\
=\sum_{i=1}^{n} \log \Phi_{2}\left(q_{T} \beta_{T}{ }^{\prime} Z_{i}, q\left(\left(1-T_{i}\right) \beta_{0}{ }^{\prime} X_{i}+T_{i} \beta_{1}{ }^{\prime} X_{i}\right), q q_{T}\left(\left(1-T_{i}\right) \rho_{T 0}+T_{i} \rho_{T 1}\right)\right)
\end{gathered}
$$

donde $\Phi_{2}(x, y, \rho)$ representa la función de densidad normal bivariante con medias cero, desviaciones estándar unitarias y correlación $\rho$, mientras que $q$ y $q_{T}$ son las variables binarias definidas por $q=2 Y-1$, donde $Y=T Y_{1}+(1-T) Y_{0}$ es la situación de empleo observada, y $q_{T}=2 T-1$.

El efecto individual $\left(\Delta_{\mathrm{i}}\right)$ de la forma de tenencia de la vivienda habitual sobre cada uno de los resultados laborales vendría determinado por $\Delta_{i}=Y_{1 i}-Y_{0 i}$. Dado que este efecto no puede ser calculado directamente a partir de los datos porque no se observa al individuo simultáneamente viviendo en alquiler y en propiedad, consideramos esta magnitud como una variable aleatoria cuya distribución de probabilidad interesa caracterizar de cara a sintetizar los efectos que genera, para la población bajo estudio, la forma de tenencia de la vivienda habitual sobre la situación de empleo/desempleo del cabeza de familia.

Por tanto, nuestro objetivo será calcular los parámetros de efectos de tratamiento medio estándar: el efecto medio del tratamiento: $\Delta^{\mathrm{ATE}}=\mathrm{E}[\Delta]=\mathrm{E}\left[Y_{1}-Y_{0}\right]$, el cual determinará el impacto medio derivado de la forma de tenencia de la vivienda habitual para un individuo seleccionado de 
la población al azar; el efecto medio del tratamiento sobre los tratados: $\Delta^{\mathrm{ATT}}=\mathrm{E}[\Delta \mid T=1]=\mathrm{E}\left[Y_{1}-Y_{0} \mid T=1\right]$, que indicará el efecto medio de la forma de tenencia sobre la situación laboral de los propietarios de su vivienda habitual; o, por último el efecto medio del tratamiento sobre los no tratados: $\Delta^{\mathrm{ATU}}=\mathrm{E}[\Delta \mid T=0]=\mathrm{E}\left[Y_{1}-Y_{0} \mid T=0\right]$, que reflejará el efecto medio de la tenencia de vivienda habitual sobre la situación laboral de los inquilinos.

Estos parámetros se derivan como en Aakvik et al (2005) a partir del modelo estructural estimado, teniéndose que, condicionados a un valor de las variables observadas $X$ y $Z$ :

$$
\begin{gathered}
\Delta^{\mathrm{ATE}}(X)=\Phi\left(\beta_{1}^{\prime} X\right)-\Phi\left(\beta_{0}^{\prime} X\right) \\
\Delta^{\mathrm{ATT}}(X, Z)=\frac{1}{\Phi\left(\beta_{T}^{\prime} Z\right)}\left[\Phi_{2}\left(\beta_{T}^{\prime} Z, \beta_{1}^{\prime} X, \rho_{T 1}\right)-\Phi_{2}\left(\beta_{T}^{\prime} Z, \beta_{0}^{\prime} X, \rho_{T 0}\right)\right] \\
\Delta^{\mathrm{ATU}}(X, Z)=\frac{1}{\Phi\left(-\beta_{T}^{\prime} Z\right)}\left[\Phi_{2}\left(-\beta_{T}^{\prime} Z, \beta_{1}^{\prime} X,-\rho_{T 1}\right)-\Phi_{2}\left(-\beta_{T}^{\prime} Z, \beta_{0}^{\prime} X,-\rho_{T 0}\right)\right]
\end{gathered}
$$

siendo $\Phi$ la función de distribución de probabilidades de la normal estándar. En consecuencia, una estimación consistente de los efectos de tratamiento medios incondicionales viene dada por las correspondientes medias muestrales, esto es:

$$
\begin{gathered}
\hat{\Delta}^{\mathrm{ATE}}=\frac{1}{n} \sum_{i=1}^{n}\left(\Phi\left(\hat{\beta}_{1}^{\prime} X_{i}\right)-\Phi\left(\hat{\beta}_{0}^{\prime} X_{i}\right)\right) \\
\hat{\Delta}^{\mathrm{ATT}}=\frac{1}{n_{1}} \sum_{i=1}^{n_{1}}\left(\frac{1}{\Phi\left(\hat{\beta}_{T}^{\prime} Z_{i}\right)}\left[\Phi_{2}\left(\hat{\beta}_{T}^{\prime} Z_{i}, \hat{\beta}_{1}^{\prime} X_{i}, \rho_{T 1}\right)-\Phi_{2}\left(\hat{\beta}_{T}^{\prime} Z_{i}, \hat{\beta}_{0}^{\prime} X_{i}, \hat{\rho}_{T 0}\right)\right]\right) \\
\left.=\frac{1}{n-n_{1}} \sum_{i=1}^{n-n_{1}}\left(\frac{1}{\Phi\left(-\hat{\beta}_{T}^{\prime} Z_{i}\right)}\left[\Phi_{2}\left(-\hat{\beta}_{T}^{\prime} Z_{i}, \hat{\beta}_{1}^{\prime} X_{i},-\hat{\rho}_{T 1}\right)-\Phi_{2}\left(-\hat{\beta}_{T}^{\prime} Z_{i}, \hat{\beta}_{0}^{\prime} X_{i},-\hat{\rho}_{T 0}\right)\right]\right)\right]
\end{gathered}
$$

donde $n_{1}$ es el número de individuos propietarios de la vivienda, y en (10) y (11) se entienden incluidos en los sumatorios exclusivamente las observaciones correspondientes a propietarios de la vivienda habitual o inquilinos, respectivamente. Los errores estándar de los distintos parámetros de efectos de tratamiento medios se pueden obtener mediante el método delta puesto que los estimadores de efectos medios de tratamiento propuestos representan funciones no lineales diferenciables respecto de los parámetros estimados para el modelo de elección discreta (1)-(4) (véase por ejemplo, Wooldridge, 2010, p. 46-47). 


\section{RESULTADOS}

En la Tabla 2 recogemos la estimación de tres modelos switching probit obtenidos cada uno para la muestra total de propietarios, propietarios con hipoteca y propietarios sin hipoteca, respectivamente, siempre confrontados con los individuos en alquiler de mercado, con objeto de poder contrastar la línea argumental de Flatau et al. (2003) y Rouwendal y Nijkamp (2010) que señala que los propietarios que poseen una hipoteca presentan menor probabilidad de estar desempleado.

Tabla 2

Modelo Switching probit de tenencia de vivienda y empleo

\begin{tabular}{|c|c|c|c|c|c|c|c|c|c|}
\hline & \multicolumn{3}{|c|}{$\begin{array}{l}\text { Propietarios } \\
\text { vs. alquiler }\end{array}$} & \multicolumn{3}{|c|}{$\begin{array}{l}\text { Propietarios con hipoteca } \\
\text { vs. alquiler }\end{array}$} & \multicolumn{3}{|c|}{$\begin{array}{l}\text { Propietarios sin hipoteca } \\
\text { vs. alquiler }\end{array}$} \\
\hline & $\begin{array}{l}\text { Tenencia } \\
\text { vivienda pro }\end{array}$ & $\begin{array}{l}\text { Empleo } \\
\text { ropietarios i }\end{array}$ & $\begin{array}{l}\text { Empleo } \\
\text { inquilinos }\end{array}$ & $\begin{array}{l}\text { Tenencia } \\
\text { vivienda p }\end{array}$ & $\begin{array}{l}\text { Empleo } \\
\text { ropietarios }\end{array}$ & $\begin{array}{l}\text { Empleo } \\
\text { inquilinos }\end{array}$ & $\begin{array}{l}\text { Tenencia } \\
\text { vivienda p }\end{array}$ & $\begin{array}{l}\text { Empleo } \\
\text { ropietarios i }\end{array}$ & $\begin{array}{l}\text { Empleo } \\
\text { inquilinos }\end{array}$ \\
\hline & Coef. & Coef. & Coef. & Coef. & Coef. & Coef. & Coef. & Coef. & Coef. \\
\hline CTE & $\begin{array}{l}-2.128^{* *} \\
(0.271)\end{array}$ & $\begin{array}{r}0.042 \\
(0.285)\end{array}$ & $\begin{array}{r}0.453 \\
(0.324)\end{array}$ & $\begin{array}{l}-2.518^{* *} \\
(0.290)\end{array}$ & $\begin{array}{l}-0.133 \\
(0.415)\end{array}$ & $\begin{array}{r}0.456 \\
(0.324)\end{array}$ & $\begin{array}{l}-3.144^{* *} \\
(0.543)\end{array}$ & $\begin{array}{r}0.255 \\
(0.414)\end{array}$ & $\begin{array}{r}0.451 \\
(0.324)\end{array}$ \\
\hline ESTU2 & $\begin{array}{r}0.047 \\
(0.076)\end{array}$ & $\begin{array}{l}0.445 \text { ** } \\
(0.056)\end{array}$ & ** $\begin{array}{r}0.153 \\
(0.142)\end{array}$ & $\begin{array}{r}0.046 \\
(0.084)\end{array}$ & $\begin{array}{l}0.466^{* *} \\
(0.087)\end{array}$ & $\begin{array}{r}0.158 \\
(0.142)\end{array}$ & $\begin{array}{r}0.085 \\
(0.110)\end{array}$ & $\begin{array}{l}0.419^{* *} \\
(0.075)\end{array}$ & $\begin{array}{r}0.142 \\
(0.142)\end{array}$ \\
\hline ESTU3 & $\begin{array}{l}0.228^{* *} \\
(0.084)\end{array}$ & $\begin{array}{l}1.055^{* \star} \\
(0.066)\end{array}$ & ** $\begin{array}{c}0.894 \\
(0.167)\end{array}$ & $\begin{array}{l}0.263^{* *} \\
(0.091)\end{array}$ & $\begin{array}{l}1.037^{* *} \\
(0.097)\end{array}$ & $\begin{array}{c}0.909^{* \star} \\
(0.167)\end{array}$ & $\begin{array}{l}0.261 * \\
(0.124)\end{array}$ & $\begin{array}{l}1.068^{* *} \\
(0.097)\end{array}$ & $\begin{array}{l}0.873^{* *} \\
(0.165)\end{array}$ \\
\hline SEXO & $\begin{array}{r}-0.064 \\
(0.057)\end{array}$ & $\begin{array}{r}0.058 \\
(0.048)\end{array}$ & $\begin{array}{l}-0.270 \\
(0.107)\end{array}$ & $\begin{array}{r}-0.090 \\
(0.061)\end{array}$ & $\begin{array}{l}0.152 * \\
(0.066)\end{array}$ & $\begin{array}{c}-0.268^{*} \\
(0.107)\end{array}$ & $\begin{array}{r}0.007 \\
(0.086)\end{array}$ & $\begin{array}{r}-0.074 \\
(0.072)\end{array}$ & $\begin{array}{l}-0.271 \text { * } \\
(0.107)\end{array}$ \\
\hline EDAD35 & $\begin{array}{l}0.669^{* *} \\
(0.244)\end{array}$ & $\begin{array}{r}-0.005 \\
(0.277)\end{array}$ & $\begin{array}{r}-0.515 \\
(0.318)\end{array}$ & $\begin{array}{l}0.817^{* *} \\
(0.259)\end{array}$ & $\begin{array}{r}0.150 \\
(0.386)\end{array}$ & $\begin{array}{r}-0.500 \\
(0.318)\end{array}$ & $\begin{array}{r}0.197 \\
(0.524)\end{array}$ & $\begin{array}{r}-0.251 \\
(0.432)\end{array}$ & $\begin{array}{l}-0.519 \\
(0.315)\end{array}$ \\
\hline EDAD45 & $\begin{array}{l}0.841^{* *} \\
(0.252)\end{array}$ & $\begin{array}{r}-0.270 \\
(0.279)\end{array}$ & $\begin{array}{l}-0.861 \\
(0.341)\end{array}$ * & $\begin{array}{l}0.953^{* *} \\
(0.269)\end{array}$ & $\begin{array}{r}-0.044 \\
(0.391)\end{array}$ & $\begin{array}{c}-0.845^{*} \\
(0.340)\end{array}$ & $\begin{array}{r}0.631 \\
(0.524)\end{array}$ & $\begin{array}{r}-0.589 \\
(0.424)\end{array}$ & $\begin{array}{l}-0.882^{* *} \\
(0.338)\end{array}$ \\
\hline EDAD55 & $\begin{array}{r}0.485 \\
(0.259)\end{array}$ & $\begin{array}{r}-0.437 \\
(0.284)\end{array}$ & $\begin{array}{l}-0.980 * * \\
(0.354)\end{array}$ & $\begin{array}{r}0.506 \\
(0.278)\end{array}$ & $\begin{array}{l}-0.195 \\
(0.397)\end{array}$ & $\begin{array}{c}-0.970^{* *} \\
(0.352)\end{array}$ & $\begin{array}{r}0.513 \\
(0.530)\end{array}$ & $\begin{array}{r}-0.653 \\
(0.427)\end{array}$ & $\begin{array}{l}-1.012^{\star *} \\
(0.354)\end{array}$ \\
\hline EDAD55M & $\begin{array}{r}0.487 \\
(0.270)\end{array}$ & $\begin{array}{l}-0.793 \text { * } \\
(0.288)\end{array}$ & ** $\begin{array}{l}-0.797 \\
(0.393)\end{array}$ & $\begin{array}{r}0.295 \\
(0.292)\end{array}$ & $\begin{array}{r}-0.385 \\
(0.412)\end{array}$ & $\begin{array}{c}-0.786^{*} \\
(0.388)\end{array}$ & $\begin{array}{r}0.704 \\
(0.539)\end{array}$ & $\begin{array}{l}-1.082 \text { * } \\
(0.431)\end{array}$ & $\begin{array}{l}-0.859 \text { * } \\
(0.396)\end{array}$ \\
\hline NTRAB & $\begin{array}{l}0.052^{* *} \\
(0.010)\end{array}$ & $\begin{array}{l}0.067 \text { ** } \\
(0.008)\end{array}$ & $* * \begin{array}{c}0.063 \\
(0.019)\end{array}$ & $\begin{array}{l}0.071^{* *} \\
(0.011)\end{array}$ & $\begin{array}{l}0.066^{* *} \\
(0.013)\end{array}$ & $\begin{array}{c}0.065^{\star *} \\
(0.019)\end{array}$ & $\begin{array}{l}0.027^{*} \\
(0.013)\end{array}$ & $\begin{array}{l}0.069^{* *} \\
(0.011)\end{array}$ & $\begin{array}{l}0.063^{* *} \\
(0.019)\end{array}$ \\
\hline NTRAB2 & $\begin{array}{l}-0.000^{* *} \\
(0.000)\end{array}$ & $\begin{array}{l}-0.000 \text { ** } \\
(0.000)\end{array}$ & $* * \begin{array}{l}-0.000 * \\
(0.000)\end{array}$ & $\begin{array}{l}-0.001^{* *} \\
(0.000)\end{array}$ & $\begin{array}{l}-0.000^{* *} \\
(0.000)\end{array}$ & $\begin{array}{c}-0.000 * \\
(0.000)\end{array}$ & $\begin{array}{r}-0.000 \\
(0.000)\end{array}$ & $\begin{array}{l}-0.000^{* *} \\
(0.000)\end{array}$ & $\begin{array}{l}-0.000 \text { * } \\
(0.000)\end{array}$ \\
\hline TPARO1 & $\begin{array}{r}0.082 \\
(0.077)\end{array}$ & $\begin{array}{l}-0.353 \text { ** } \\
(0.059)\end{array}$ & $\begin{array}{r}-0.098 \\
(0.157)\end{array}$ & $\begin{array}{r}0.087 \\
(0.084)\end{array}$ & $\begin{array}{l}-0.315^{\star *} \\
(0.084)\end{array}$ & $\begin{array}{l}-0.092 \\
(0.156)\end{array}$ & $\begin{array}{r}0.099 \\
(0.111)\end{array}$ & $\begin{array}{l}-0.374^{* *} \\
(0.085)\end{array}$ & $\begin{array}{l}-0.112 \\
(0.157)\end{array}$ \\
\hline TPARO2 & $\begin{array}{l}-0.162 \text { * } \\
(0.071)\end{array}$ & $\begin{array}{r}-0.092 \\
(0.055)\end{array}$ & $\begin{array}{r}0.062 \\
(0.129)\end{array}$ & $\begin{array}{l}-0.171^{*} \\
(0.076)\end{array}$ & $\begin{array}{r}-0.075 \\
(0.079)\end{array}$ & $\begin{array}{r}0.058 \\
(0.129)\end{array}$ & $\begin{array}{r}-0.159 \\
(0.104)\end{array}$ & $\begin{array}{r}-0.105 \\
(0.078)\end{array}$ & $\begin{array}{r}0.064 \\
(0.128)\end{array}$ \\
\hline PAREJA & $\begin{array}{l}0.4311^{* *} \\
(0.072)\end{array}$ & - & - & $\begin{array}{l}0.554^{* *} \\
(0.079)\end{array}$ & - & - & $\begin{array}{r}0.144 \\
(0.102)\end{array}$ & - & - \\
\hline MIEMB & $\begin{array}{c}0.121 \\
(0.062)\end{array}$ & - & - & $\begin{array}{r}0.081 \\
(0.072)\end{array}$ & - & - & $\begin{array}{l}0.262^{\star *} \\
(0.089)\end{array}$ & - & - \\
\hline MIEMB2 & $\begin{array}{l}-0.017 \text { * } \\
(0.007)\end{array}$ & - & - & $\begin{array}{r}-0.012 \\
(0.008)\end{array}$ & - & - & $\begin{array}{l}-0.032^{* *} \\
(0.011)\end{array}$ & - & - \\
\hline LPRELAT & $\begin{array}{l}0.166 \text { * } \\
(0.081)\end{array}$ & - & - & $\begin{array}{l}0.209 * \\
(0.089)\end{array}$ & - & - & $\begin{array}{r}0.127 \\
(0.114)\end{array}$ & - & - \\
\hline CCAA1 & $\begin{array}{r}-0.098 \\
(0.069)\end{array}$ & - & - & $\begin{array}{r}-0.048 \\
(0.074)\end{array}$ & - & - & $\begin{array}{r}-0.185 \\
(0.098)\end{array}$ & - & - \\
\hline CCAA2 & $\begin{array}{l}-0.155 \text { * } \\
(0.077)\end{array}$ & - & - & $\begin{array}{r}-0.159 \\
(0.084)\end{array}$ & - & - & $\begin{array}{r}-0.197 \\
(0.109)\end{array}$ & - & - \\
\hline NACIDO & $\begin{array}{l}1.312^{* *} \\
(0.066)\end{array}$ & - & - & $\begin{array}{l}1.304^{* *} \\
(0.069)\end{array}$ & - & - & $\begin{array}{l}1.490^{* *} \\
(0.109)\end{array}$ & - & - \\
\hline
\end{tabular}


Tabla 2 (continuación)

Modelo Switching probit de tenencia de vivienda y empleo

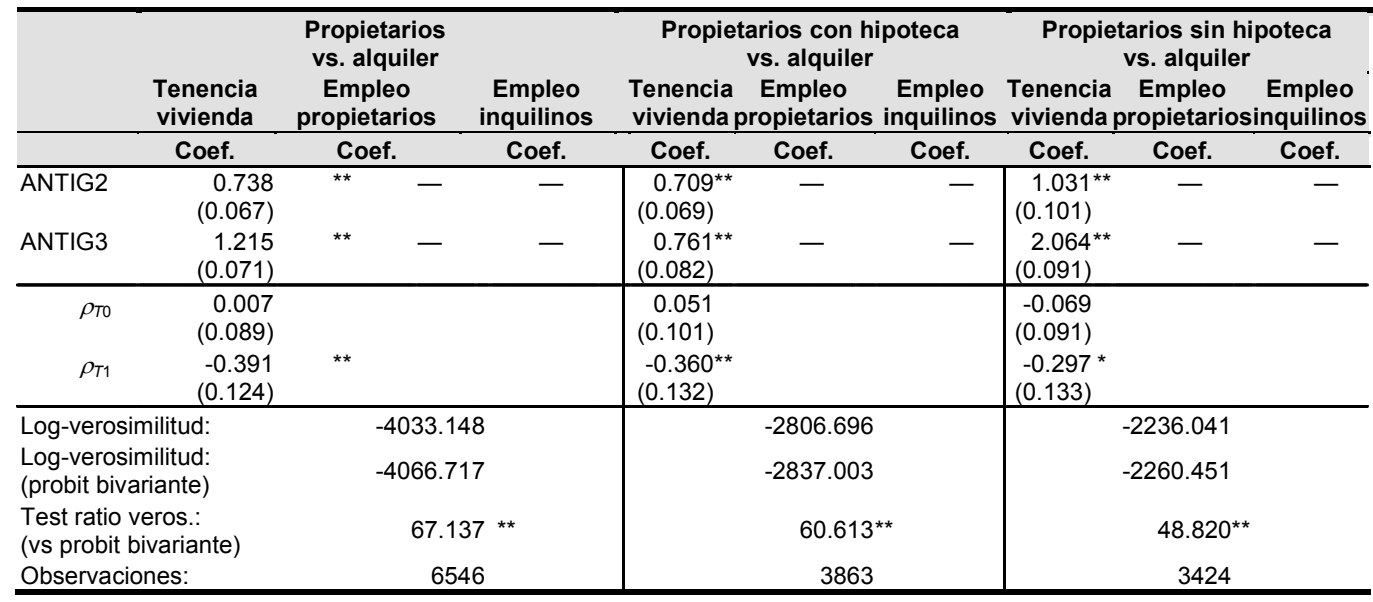

Nota: Error estándar entre paréntesis. * significatividad al 5\%, ** significatividad al $1 \%$.

Fuente: Elaboración propia.

Los valores obtenidos para las tres muestras resultan muy similares, habiéndose comprobado mediante tests de Wald la significatividad conjunta de las variables multinomiales incluidas (edad, estudios, y CC.AA. por tasas de paro y por niveles de precio de la vivienda). En todos los casos se evidencia la existencia de diferencias en la situación de empleo entre propietarios e inquilinos, al validarse el modelo switching probit frente al probit bivariante según el test de razón de verosimilitudes. En general, se evidencia que el poseer un mayor nivel de estudios, disponer de un mayor número de años pasados en trabajo remunerado y ubicar su residencia en comunidades autónomas con menores niveles de desempleo, son todos ellos factores que incrementan significativamente la probabilidad de empleo para los propietarios de la vivienda, al igual que el hecho de ser varón en la muestra de propietarios con hipoteca, mientras que poseer más de 55 años incide de forma negativa cuando se considera el total de propietarios y especialmente la muestra de propietarios sin hipoteca.

En cambio, los principales determinantes en la situación de empleo para los inquilinos son el ser joven, mujer y poseer mayores niveles de estudio y años trabajados con anterioridad.

Por otro lado, la estimación de las ecuaciones de tenencia de vivienda indican que los hogares con una mayor antigüedad en la vivienda actual, donde el cabeza de familia vive en pareja, ha nacido en España, y posee un mayor nivel de estudios, edad y número de años trabajados, son los que muestran mayor probabilidad de tener su vivienda habitual en propiedad. Estos resultados resul- 
tan similares a los obtenidos en Barrios y Rodríguez (2005) o Rodríguez y Barrios (2007).

De igual forma, la significatividad del coeficiente de correlación $\rho_{T 1}$ del modelo switching probit evidencia la interrelación entre ambos mercados ${ }^{7}$, indicando que los factores inobservados que promueven la decisión de vivienda en propiedad desincentivan la situación de empleo para los propietarios.

En la Tabla 3 recogemos la estimación de los parámetros de efectos medios del tratamiento que resultan de los modelos switching probit calculados para tres muestras diferentes: propietarios vs alquiler de mercado, propietarios con hipoteca vs alquiler de mercado, y propietarios sin hipoteca vs alquiler de mercado.

Tabla 3

Efectos medios de la forma de tenencia de la vivienda habitual sobre el empleo

\begin{tabular}{cccc}
\hline & I & II & III \\
\hline Efecto & Estimación & Estimación & Estimación \\
\hline$\Delta^{\text {ATE }}$ & $0.113^{* *}$ & $0.118^{* *}$ & $0.116^{* *}$ \\
& $(0.035)$ & $(0.034)^{* *}$ & $(0.030)$ \\
$\Delta^{\text {ATT }}$ & $0.102^{* *}$ & $0.102^{* *}$ & $0.111^{* *}$ \\
& $(0.036)^{* *}$ & $(0.034)^{* *}$ & $(0.033)^{* *}$ \\
$\Delta^{\text {ATU }}$ & $0.190^{* *}$ & $0.187^{* *}$ & $0.133^{* *}$ \\
& $(0.034)$ & $(0.034)$ & $(0.025)$ \\
\hline
\end{tabular}

Nota: I: Propietarios vs alquiler de mercado, II: Propietarios con hipoteca vs alquiler de mercado; III: Propietarios sin hipoteca vs alquiler de mercado. Errores estándar entre paréntesis. * significativo al $5 \%$, ** significativo al $1 \%$.

Fuente: Elaboración propia.

Los resultados alcanzados muestran un efecto medio positivo de la vivienda en propiedad sobre la probabilidad de empleo en las tres muestras consideradas, donde los parámetros ATE, ATT y ATU resultan significativamente distintos de cero al $1 \%$ de significatividad, obteniéndose para este último parámetro valores notablemente superiores que deberán ser considerados con la debida cautela dado el reducido tamaño de la muestra de alquiler (741 individuos). En concreto, para la muestra I, se estima que la probabilidad de empleo aumenta por término medio, cuando consideramos que el individuo reside en una vivienda en propiedad frente a una en alquiler, en un $11.3 \%$ para toda la población, un $10.2 \%$ para el caso de los propietarios y un $19 \%$ para los inquilinos. Estos valores alcanzados para la muestra I resultan muy similares a los obtenidos para la muestra II y III (salvo el parámetro ATU en la muestra III). En consecuencia,

\footnotetext{
${ }^{7}$ Se comprueba también mediante un test de Wald la significatividad conjunta de ambos coeficientes de correlación $\left(\rho_{T 1}, \rho_{T 2}\right)$ para las tres muestras.
} 
según estos resultados cabría a priori pensar que el hecho de disponer de una hipoteca no parece generar entre los propietarios de la vivienda un comportamiento diferenciado en términos de empleo, contrariamente a la línea argumental que señala Rouwendal y Nijkamp (2010) o Flatau et al. (2003).

Esta incidencia positiva de la tenencia de vivienda en propiedad sobre el empleo podría venir explicada por el "efecto vecindario" que señala Ioannides y Loury (2004). Estos autores argumentan que las redes de información de empleo que se generan en el vecindario favorecen la búsqueda de trabajo. En ese sentido, el mejor conocimiento y grado de implicación con el vecindario que poseen los propietarios como consecuencia de su mayor permanencia por término medio en la zona, puede hacer que este colectivo tenga más facilidad que los inquilinos para encontrar un nuevo empleo ${ }^{8}$. Para contrastar la tesis de Ioannides y Loury (2004), subdividimos la muestra en base a los años de antigüedad en la vivienda y estimamos para cada una de las nuevas submuestras, los correspondientes parámetros de efectos medios del tratamiento bajo el uso de modelos switching probit. En este sentido, dado que nuestro interés es analizar el papel que desempeña la tenencia de hipoteca y la antigüedad en la vivienda como posibles moduladores de la influencia del mercado de vivienda sobre el mercado de trabajo, en la Tabla 4 se presentan los valores obtenidos para la muestra I y II, realizando la subdivisión para una antigüedad de 4 años ${ }^{9}$.

Según los datos recogidos en la Tabla 4, el hecho de que todas las estimaciones de los efectos medios de tratamiento resulten superiores en la submuestra que incluye a los individuos con una antigüedad en la vivienda superior a 4 años (salvo el parámetro ATE estimado para el total de propietarios que muestra idéntico valor en ambas submuestras), parece evidenciar que una mayor antigüedad en la vivienda desempeña un efecto positivo en la probabilidad de estar empleado en ambas muestras (Muestra I y Muestra II). Este resultado confirmaría la hipótesis de Ioannides y Loury (2004). Por otro lado, si comparamos los resultados de la muestra I y II, observamos que para ambas submuestras los propietarios con hipoteca son los que presentan mayores valores en los efectos medios del tratamiento ATE y ATT, con una estimación del parámetro ATE para una antigüedad inferior y superior a 4 años de 0.14 y 0.15 , respectivamente. Valores estos que resultan claramente superiores a los de la muestra I y a los obtenidos en la Tabla 3. Asimismo, dentro de la muestra de propietarios con

\footnotetext{
${ }^{8}$ A este respecto es significativo que un estudio de la EPA de 2009 elaborado por el INE dilucidara que casi la mitad de los jóvenes españoles $(47,9 \%)$ encontraron su primer empleo gracias a un familiar o amigo.

${ }^{9}$ También se ha subdividido la muestra atendiendo a distintos períodos de antigüedad, siendo los resultados similares a los mostrados en la Tabla 4, teniéndose no obstante problemas de estimación en algunos casos debido al reducido número de individuos existentes en algunas submuestras.
} 
hipoteca, los que poseen una mayor antigüedad en la vivienda actual son los que muestran estimaciones superiores en los parámetros ATE y ATT. Por tanto, estos resultados parecen indicar que tanto la antigüedad como la hipoteca resultan ser factores que explican la incidencia positiva de la tenencia en propiedad sobre el empleo. La influencia simultánea de estos dos factores permite responder al interrogante de por qué los propietarios sin hipoteca muestran menores parámetros de efectos de tratamiento a pesar de poseer una mayor antigüedad en la vivienda ${ }^{10}$ y un mayor número de años pasados en trabajo remunerado ${ }^{11}$. Variables estas últimas que inciden positivamente y de forma muy relevante en la ecuación de empleo.

\section{Tabla 4}

Estimación de los efectos medios del tratamiento según antigüedad en la vivienda

\begin{tabular}{ccc|cc}
\hline & \multicolumn{2}{c|}{ Muestra I } & \multicolumn{2}{c}{ Muestra II } \\
\hline E.T. I Antigüedad: & $\mathbf{5 4 \text { años }}$ & $\mathbf{> 4 \text { años }}$ & $\mathbf{5 4 \text { años }}$ & $\mathbf{7}$ años \\
\hline$\Delta^{\text {ATE }}$ & $0.128^{* *}$ & $0.128^{*}$ & $0.141^{* *}$ & $0.150^{*}$ \\
& $(0.046)$ & $(0.072)$ & $(0.046)$ & $(0.083)$ \\
$\Delta^{\text {ATT }}$ & $0.082^{*}$ & $0.120^{*}$ & $0.089^{*}$ & $0.138^{*}$ \\
$\Delta^{\text {ATU }}$ & $(0.047)$ & $(0.072)$ & $(0.046)$ & $(0.080)$ \\
& $0.202^{* *}$ & $0.264^{* *}$ & $0.217^{* *}$ & $0.251^{* *}$ \\
& $(0.049)$ & $(0.076)$ & $(0.050)$ & $(0.078)$ \\
\hline Total Observaciones: & 1167 & 5379 & 1079 & 2784 \\
\hline
\end{tabular}

Nota: I: Propietarios vs alquiler de mercado, II: Propietarios con hipoteca vs alquiler de mercado. Errores estándar entre paréntesis. * significativo al 10\%, ** significativo al $5 \%$.

Fuente: Elaboración propia.

\section{CONCLUSIONES}

El objetivo de este trabajo es aportar alguna evidencia empírica sobre la posible existencia de relaciones causales entre el régimen de tenencia de la vivienda (propiedad/alquiler) y la situación en el empleo (empleado/desempleado) mediante el uso de los microdatos anuales de la Encuesta de Condiciones de Vida para el periodo 2010. ). Para ello, adoptamos el enfoque de la literatura sobre efectos de un tratamiento, el cual nos permite separar este efecto del generado por otros covariantes e inobservables que puedan afectar. Además, para evitar el problema de la causalidad inversa, se incluyen en la muestra solo aquellos hogares que han permanecido en la vivienda actual al menos durante los dos últimos años 2009 y 2010, garantizándose así que la situación actual de empleo no ha influido en la decisión de tenencia de la vivienda actual.

\footnotetext{
${ }^{10}$ Este colectivo tienen una antigüedad media en la vivienda de 22 años, frente a los 9.3 años que tiene los propietarios con hipoteca.

${ }^{11}$ Véase los estadísticos descriptivos recogidos en la Tabla A2.
} 
En primer lugar, se han estimado modelos switching probit para tres muestras diferentes: propietarios vs alquiler de mercado, propietarios con hipoteca vs alquiler de mercado, y propietarios sin hipoteca vs alquiler de mercado, con objeto de poder contrastar si la existencia de hipoteca conlleva diferencias en términos de empleo. Los resultados obtenidos para estas tres muestras son similares, poniéndose de manifiesto en todo caso la existencia de diferencias en la situación de empleo entre los propietarios e inquilinos. En concreto, los factores que incrementan significativamente la probabilidad de empleo para los propietarios de la vivienda son el poseer un mayor nivel de estudios, disponer de un mayor número de años pasados en trabajo remunerado y ubicar su residencia en comunidades autónomas con menores niveles de desempleo, así como ser varón en la muestra de propietarios con hipoteca. Para lo inquilinos, los principales determinantes de la situación de empleo son el ser joven, mujer y poseer mayores niveles de estudio y años trabajados.

Por otro lado, la estimación de la ecuación de tenencia de vivienda resulta similar a la obtenida por Barrios y Rodríguez (2005) o Rodríguez y Barrios (2007), indicando que los hogares con una mayor antigüedad en la vivienda actual, donde el cabeza de familia vive en pareja, ha nacido en España, y posee un mayor nivel de estudios, edad y número de años trabajados, son los que muestran mayor probabilidad de tener su vivienda habitual en propiedad. Además se corrobora la existencia de interrelación entre los mercados de vivienda y de trabajo a través de la significatividad del coeficiente de correlación $\rho_{T 1}$, indicando que los factores inobservados que promueven la decisión de vivienda en propiedad desincentivan la situación de empleo para los propietarios.

En segundo lugar, se han estimado los parámetros de efectos medios del tratamiento, evidenciándose un efecto medio positivo de la vivienda en propiedad sobre la probabilidad de empleo en las tres muestras consideradas. En concreto, para la muestra de propietarios vs alquiler de mercado se estima que la probabilidad de empleo aumenta por término medio en un $11.3 \%$ para toda la población, un $10.2 \%$ para el caso de los propietarios, incrementándose esta probabilidad hasta un $19 \%$ si nos circunscribimos a los inquilinos. Por tanto, se pone de manifiesto que en España la tenencia de vivienda en propiedad genera un efecto positivo sobre el empleo, contrariamente a la tesis de Oswald (1996). En ese sentido, se confirman las conclusiones obtenidas por Barrios y Rodríguez (2004) a nivel macroeconómico, pero se evidencia un efecto superior a nivel microeconómico y en torno a 10-11 puntos porcentuales de incremento en la probabilidad de empleo para los propietarios de la vivienda habitual.

Por último, para profundizar en el análisis, subdividimos la muestra en base a los años de antigüedad en la vivienda y estimamos los parámetros de efectos medios del tratamiento haciendo uso de modelos switching probit. Los valores estimados indican que una mayor antigüedad en la vivienda y el poseer una 
hipoteca resultan ser factores que de forma conjunta explican la incidencia positiva de la tenencia en propiedad sobre el empleo. Una de las causas que explicarían este resultado la encontramos, según señala Ioannides y Loury (2004), en que los propietarios, al tener un mayor grado de implicación con la zona donde residen, pueden beneficiarse en mayor medida de las redes de información que se generan en el vecindario y que favorecen la búsqueda de empleo. Por otro lado, los propietarios con hipoteca que han perdido su trabajo se ven obligados a desempeñar una mayor intensidad en la búsqueda de un empleo para poder hacer frente al pago de la hipoteca y no perder la vivienda que habitan.

Una importante consecuencia del análisis realizado es que intervenir el mercado de vivienda con objeto de favorecer el acceso a una vivienda en propiedad no resulta incompatible con el objetivo de reducción del desempleo. De forma paralela, la incentivación del alquiler tampoco parece una medida acertada en términos únicamente de fomento de empleo.

\section{REFERENCIAS BIBLIOGRÁFICAS}

AAKVIK, A.; HECKMAN, J. J. y VYTLACIL, E. J. (2005). "Estimating treatment effects for discrete outcomes when responses to treatment vary: an application to Norwegian vocational rehabilitation programs". Journal of Econometrics, 125 , pp. 15-51.

BARCELÓ, C. (2006). "Housing tenure and labour mobility: a comparison across European countries". Documentos de Trabajo del Banco de España $\mathrm{n}^{\circ}$ 0603, Madrid.

BARRIOS, J. A. y RODRÍGUEZ, J. E. (2004). "User cost changes, unemployment and home-ownership: evidence from Spain”. Urban Studies, 41 (3), pp. 563-578.

BARRIOS, J. A. y RODRÍGUEZ, J. E. (2005). "Un modelo logit multinomial mixto de tenencia de vivienda". Revista de Economía Aplicada, 13 (38), pp. 5-27.

BENTOLILA, S. y BLANCHARD, O. J. (1990). "Spanish unemployment". Economic Policy, 10, pp. 234-281.

BLANCHFLOWER, D. G. y OSWALD. A. J. (1994). "The wage curve". Cambridge, MA: MIT Press.

BÖHEIM, R. y TAYLOR, M. P. (2002). "Tied down or room to move? Investigating the relationships between housing tenure, employment status and reside". Scottish Journal of Political Economy, 49 (4), pp. 369-392. 
BOVER, O.; MUELLBAUER, J. y MURPHY, A. (1989). "House prices, wages and the UK labour market". Oxford Bulletin of Economics and Statistics, 51, pp. 97-136.

BRUNET, C. y LESUEUR, J. Y. (2009). "Do homeowners stay unemployed longer? A French microeconometric study". En Van Ewijk, C. y Van Leuvenjstein, M. (eds.), Homeownership and the labour market in Europe (pp. 137-160). New York: Oxford University Press.

CARRASCO, R. (2001). "Binary choice with binary endogenous regressors in panel data: estimating the effect of fertility on female labor participation". Journal of Business and Economic Statistics, 19 (4), pp. 385-394.

COULSON, N. E. y FISHER, L. M. (2002). "Tenure choice and labour market outcomes". Housing Studies, 17, pp. 35-49.

COULSON, N. E. y FISHER, L. M. (2009). "Housing tenure and labor market impacts: the search goes on". Journal of Urban Economics, 65 (3), pp. 252264.

DOHMEN, T. J. (2005). "Housing mobility and unemployment". Regional Science and Urban Economics, 35, pp. 305-325.

FLATAU, P.; FORBES, M.; HENDERSHOTT, P. H. y WOOD, G. (2003). "Homeownership and unemployment: The roles of leverage and public housing". NBER Working paper 10021, Cambridge, MA.

GARCíA, C. (2012). "Del pasmo al marasmo: El sector de la construcción y su relación con la crisis del empleo". Estudios de Economía Aplicada, 30 (1), pp. 163-182.

GREEN, R. K. y HENDERSHOTT, P. H. (2003). "Home ownership and duration of unemployment a test of the Oswald Hypothesis". CULER working paper 03-07, University of Wisconsin-Madison.

HECKMAN, J. J.; LALONDE, R. J. y SMITH, J. A. (1999). "The economics and econometrics of active labor programs". En Ashenfelter, O. C. and Card, D. (eds.), Handbook of Labor Economics Vol. 3. Amsterdam: North Holland.

HECKMAN, J. J.; LOCHNER, L. y TABER, C. (1998). "General equilibrium treatment effects: a study of tuition policy". American Economic Review, 88 (2), pp. 381-386.

HECKMAN, J. J.; TOBIAS, J. L. y VYTLACIL, E. (2003). "Simple estimators for treatment parameters in a latent-variable framework". The Review of Economics and Statistics, 85 (3), pp. 748-755.

HENLEY, A. (1998). "Residential mobility, housing equity and the labour market". The Economic Journal, 108, pp. 414-427.

HUGHES, G. y MCCORMICK, B. (1987). "Housing markets, unemployment and labour market flexibility in the UK". European Economic Review, 31, pp. 615645.

IOANNIDES, Y. M. y LOURY L. D. (2004). "Job information networks, neighborhood effects, and inequality". Journal of Economic Literature, XLII, pp. 1056-1093. 
LAYARD, R. N.; NICKELL, S. J. y JACKMAN, R. (1991). "Unemployment: Macroeconomic performance and the labour market". Oxford: Oxford University Press.

MUNCH, J. R.; ROSHOLM, M. y SVARER, M. (2006). "Are homeowners really more unemployed?". The Economic Journal, 116, pp. 991-1013.

MUNCH, J. R.; ROSHOLM, M. y SVARER, M. (2008). “Home ownership, job duration and wages". Journal of Urban Economics, 63, pp. 130-145.

NICKELL, S. (1998). "Unemployment: questions and some answers". The Economic Journal, 108, pp. 802-816.

O'HIGGINS, N. (1994). "YTS, employment, and sample selection bias". Oxford Economic Papers, 46, pp. 605-628.

OSWALD, A. J. (1996). "A conjecture on the explanation for high unemployment in the industrialized nations: Part I". Warwick Economics Research Paper, \#475, University of Warwick.

OSWALD, A. J. (1997). A theory of homes and jobs, mimeo, unpublished manuscript. University of Warwick.

PHELPS, E. S. (1994). "Structural slumps: the modern theory of unemployment, interest, and assets". Cambridge, MA: Harvard University Press.

PISSARIDES, C. A. (2000). "Equilibrium unemployment theory". Cambridge: MIT Press.

RIVERS, D. y VUONG, Q. H. (1988), "Limited information estimators and exogeneity tests for simultaneous probit models". Journal of Econometrics, 39, pp. 347-366.

RODRÍGUEZ, J. E. y BARRIOS, J. A. (2007). "Estimación microeconométrica de la tenencia y demanda de vivienda en España según la localización". Estudios de Economía Aplicada, 25 (1), pp. 453-484.

ROUWENDAL, J. y NIJKAMP, P. (2010). "Homeownership and labour market behaviour: interpreting the evidence". Environment and Planning A, 42 (2), pp. 419-433.

VAN LEUVENJSTEIN, M. y KONING, P. (2004). "The effect of home-ownership on labor mobility in the Netherlands". Journal of Urban Economics, 55, pp. 580-596.

VAN EWIJK, C. y VAN LEUVENJSTEIN, M. (2009). "Homeownership and the labour market in Europe". New York: Oxford University Press.

WOOLDRIDGE, J. M. (2010). Econometric analysis of cross section and panel data, $2^{\mathrm{a}}$ Ed., Cambridge MA: The MIT Press. 


\section{APÉNDICE}

\section{Tabla A1}

Descripción de las variables explicativas

\begin{tabular}{|c|c|}
\hline VARIABLES & DEFINICIÓN \\
\hline \multicolumn{2}{|c|}{ Características del hogar: } \\
\hline \multirow{2}{*}{$\begin{array}{r}\text { MIEMB, MIEMB2 } \\
\text { Antigüedad en la vivienda: } \\
A N T I G 1^{*}\end{array}$} & Número de miembros del hogar y su cuadrado. \\
\hline & La vivienda lleva ocupada menos de 5 años (inclusive) $=1$; resto $=0$. \\
\hline ANTIG2 & La vivienda lleva ocupada entre 6 y 10 años (inclusive) $=1$; resto $=0$. \\
\hline ANTIG3 & La vivienda lleva ocupada más de 10 años $=1$; resto $=0$. \\
\hline \multicolumn{2}{|c|}{ Características del cabeza de familia: } \\
\hline SEXO & Cabeza de familia mujer=0; cabeza de familia varón=1. \\
\hline \multirow{2}{*}{$\begin{array}{l}\text { Estado civil: } \\
\text { PAREJA }\end{array}$} & \\
\hline & Cabeza de familia vive con su pareja de hecho (con o sin base jurídica)=1; resto=0. \\
\hline \multicolumn{2}{|l|}{ Edad: } \\
\hline$E D A D 25^{*}$ & Cabeza de familia con menos de 25 años $=1$; resto $=0$. \\
\hline EDAD35 & Cabeza de familia con 25 años o más y menos de 35 años $=1$; resto $=0$. \\
\hline$E D A D 45$ & Cabeza de familia con 35 años o más y menos de 45 años $=1$; resto $=0$. \\
\hline EDAD55 & Cabeza de familia con 45 años o más y menos de 55 años $=1$; resto $=0$. \\
\hline \multirow{2}{*}{$\begin{array}{l}\text { EDAD55M } \\
\text { Estudios: }\end{array}$} & Cabeza de familia con más de 55 años $=1$; resto $=0$. \\
\hline & \\
\hline ESTU1* & Cabeza de familia sin estudios o con estudios primarios $=1$; resto $=0$. \\
\hline ESTU2 & $\begin{array}{l}\text { Cabeza de familia con estudios de educación secundaria }\left(1^{\mathrm{a}} \text { y } 2^{\mathrm{a}} \text { etapa) y formación }\right. \\
\text { e inserción laboral que precisa título de segunda etapa de secundaria=1; resto }=0 \text {. }\end{array}$ \\
\hline \multirow{2}{*}{$\begin{array}{r}E S T U 3 \\
\text { País de nacimiento: }\end{array}$} & Cabeza de familia con estudios de educación superior $=1$; resto $=0$. \\
\hline & \\
\hline $\begin{array}{r}\text { NACIDO } \\
\text { Años trabaiados. }\end{array}$ & Cabeza de familia nacido en España=1, resto $=0$. \\
\hline \multirow{2}{*}{$\begin{array}{l}\text { Años trabajados: } \\
\text { NTRAB, NTRAB2 }\end{array}$} & Nhimprga \\
\hline & $\begin{array}{l}\text { Número de años pasados en trabajo remunerado del cabeza de familia, y su } \\
\text { cuadrado. }\end{array}$ \\
\hline \multicolumn{2}{|c|}{ Otras características económicas: } \\
\hline LPRELAT & $\begin{array}{l}\text { Diferencia entre el índice de precios hedónicos de la vivienda en propiedad y en } \\
\text { alquiler (ambos en logaritmos) para la CC.AA. de residencia. }\end{array}$ \\
\hline \multirow{2}{*}{$\begin{array}{l}\text { CC.AA. según precios de } \\
\text { la vivienda }{ }^{1}: C C A A 1\end{array}$} & \\
\hline & $\begin{array}{l}\text { Comunidades con precios superiores a la media nacional (Baleares, Madrid, } \\
\text { Cantabria, Cataluña y País Vasco) }=1 \text {; resto }=0 \text {. }\end{array}$ \\
\hline CCAA2 & $\begin{array}{l}\text { Comunidades con precios entre el } 80-100 \% \text { de la media nacional (Andalucía, } \\
\text { Aragón, Asturias, Canarias, Navarra, Rioja y Ceuta y Melilla) }=1 \text {; resto }=0 \text {. }\end{array}$ \\
\hline$C C A A 3^{*}$ & $\begin{array}{l}\text { Comunidades con precios menores al } 80 \% \text { de la media nacional (Galicia, Castilla- } \\
\text { Mancha, Castilla León, C. Valenciana, Extremadura y Murcia) }=1 \text {; resto }=0 \text {. }\end{array}$ \\
\hline \multirow{2}{*}{$\begin{array}{l}\text { CC.AA. según niveles de } \\
\text { desempleo }{ }^{2}: T P A R O 1\end{array}$} & \\
\hline & $\begin{array}{l}\text { Comunidades con mayores tasas de paro en } 2009 \text { (Andalucía, Ceuta y Melilla, } \\
\text { Canarias y C. Valenciana) }=1 \text {; resto }=0 \text {. }\end{array}$ \\
\hline TPARO2 & $\begin{array}{l}\text { Comunidades con tasas de paro intermedias en } 2009 \text { (Asturias, Baleares, Murcia, } \\
\text { Castilla-León, Castilla-Mancha, Cataluña, Extremadura y Madrid)=1; resto=0. }\end{array}$ \\
\hline TPARO3* & $\begin{array}{l}\text { Comunidades con menores tasas de paro en } 2009 \text { (Aragón, Rioja, Cantabria, } \\
\text { Galicia, Navarra y País vasco) }=1 \text {; resto }=0 \text {. }\end{array}$ \\
\hline
\end{tabular}

\footnotetext{
* Variable de referencia

${ }^{1}$ Atendiendo al índice del precio de la vivienda libre elaborado por el Ministerio de Vivienda para el año 2010.

${ }^{2}$ Según datos de la Encuesta de Población Activa (E.P.A.).
}

Fuente: Elaboración propia a partir de los datos procedentes de la Encuesta de Condiciones de Vida para el año 2010 (I.N.E.) 
Tabla A2

Estadísticos descriptivos de las variables explicativas

\begin{tabular}{|c|c|c|c|c|c|}
\hline & Total & Propietarios & Inquilinos & $\begin{array}{c}\text { Propietarios con } \\
\text { hipoteca }\end{array}$ & $\begin{array}{c}\text { Propietarios sin } \\
\text { hipoteca }\end{array}$ \\
\hline ESTU1 & $\begin{array}{r}0.157 \\
(0.364)\end{array}$ & $\begin{array}{r}0.156 \\
(0.363)\end{array}$ & $\begin{array}{r}0.167 \\
(0.373)\end{array}$ & $\begin{array}{r}0.108 \\
(0.311)\end{array}$ & $\begin{array}{r}0.212 \\
(0.408)\end{array}$ \\
\hline ESTU2 & $\begin{array}{r}0.486 \\
(0.499)\end{array}$ & $\begin{array}{r}0.481 \\
(0.499)\end{array}$ & $\begin{array}{r}0.526 \\
(0.499)\end{array}$ & $\begin{array}{r}0.487 \\
(0.499)\end{array}$ & $\begin{array}{r}0.474 \\
(0.499)\end{array}$ \\
\hline ESTU3 & $\begin{array}{r}0.355 \\
(0.478)\end{array}$ & $\begin{array}{r}0.361 \\
(0.480)\end{array}$ & $\begin{array}{r}0.306 \\
(0.461)\end{array}$ & $\begin{array}{r}0.403 \\
(0.490)\end{array}$ & $\begin{array}{r}0.313 \\
(0.463)\end{array}$ \\
\hline SEXO & $\begin{array}{r}0.643 \\
(0.479)\end{array}$ & $\begin{array}{r}0.651 \\
(0.476)\end{array}$ & $\begin{array}{r}0.577 \\
(0.494)\end{array}$ & $\begin{array}{r}0.639 \\
(0.480\end{array}$ & $\begin{array}{r}0.666 \\
(0.471)\end{array}$ \\
\hline EDAD25 & $\begin{array}{r}0.007 \\
(0.084)\end{array}$ & $\begin{array}{r}0.003 \\
(0.060)\end{array}$ & $\begin{array}{r}0.035 \\
(0.184)\end{array}$ & $\begin{array}{r}0.002 \\
(0.053)\end{array}$ & $\begin{array}{r}0.004 \\
(0.066)\end{array}$ \\
\hline EDAD35 & $\begin{array}{r}0.135 \\
(0.342)\end{array}$ & $\begin{array}{r}0.116 \\
(0.320)\end{array}$ & $\begin{array}{r}0.288 \\
(0.453)\end{array}$ & $\begin{array}{r}0.180 \\
(0.385)\end{array}$ & $\begin{array}{r}0.040 \\
(0.198)\end{array}$ \\
\hline EDAD45 & $\begin{array}{r}0.306 \\
(0.461)\end{array}$ & $\begin{array}{r}0.309 \\
(0.462)\end{array}$ & $\begin{array}{r}0.288 \\
(0.453)\end{array}$ & $\begin{array}{r}0.417 \\
(0.493)\end{array}$ & $\begin{array}{r}0.183 \\
(0.386)\end{array}$ \\
\hline EDAD55 & $\begin{array}{r}0.335 \\
(0.472)\end{array}$ & $\begin{array}{r}0.343 \\
(0.474)\end{array}$ & $\begin{array}{r}0.269 \\
(0.444)\end{array}$ & $\begin{array}{r}0.295 \\
(0.456)\end{array}$ & $\begin{array}{r}0.399 \\
(0.489)\end{array}$ \\
\hline EDAD55M & $\begin{array}{r}0.214 \\
(0.410)\end{array}$ & $\begin{array}{r}0.227 \\
(0.419)\end{array}$ & $\begin{array}{r}0.117 \\
(0.322)\end{array}$ & $\begin{array}{r}0.102 \\
(0.303)\end{array}$ & $\begin{array}{r}0.372 \\
(0.483)\end{array}$ \\
\hline PAREJA & $\begin{array}{r}0.743 \\
(0.436)\end{array}$ & $\begin{array}{r}0.765 \\
(0.424)\end{array}$ & $\begin{array}{r}0.573 \\
(0.494)\end{array}$ & $\begin{array}{r}0.793 \\
(0.404)\end{array}$ & $\begin{array}{r}0.732 \\
(0.442)\end{array}$ \\
\hline MIEMB & $\begin{array}{r}3.081 \\
(1.267)\end{array}$ & $\begin{array}{r}3.107 \\
(1.223)\end{array}$ & $\begin{array}{r}2.875 \\
(1.559)\end{array}$ & $\begin{array}{r}3.081 \\
(1.212)\end{array}$ & $\begin{array}{r}3.138 \\
(1.234)\end{array}$ \\
\hline MIEMB2 & $\begin{array}{l}11.101 \\
(8.722)\end{array}$ & $\begin{array}{l}11.153 \\
(8.163)\end{array}$ & $\begin{array}{r}10.700 \\
(12.245)\end{array}$ & $\begin{array}{l}10.962 \\
(7.966)\end{array}$ & $\begin{array}{l}11.375 \\
(8.383)\end{array}$ \\
\hline NACIDO & $\begin{array}{r}0.902 \\
(0.269)\end{array}$ & $\begin{array}{r}0.948 \\
(0.221)\end{array}$ & $\begin{array}{r}0.542 \\
(0.498)\end{array}$ & $\begin{array}{r}0.924 \\
(0.264)\end{array}$ & $\begin{array}{r}0.976 \\
(0.150)\end{array}$ \\
\hline NTRAB & $\begin{array}{r}23.292 \\
(11.280)\end{array}$ & $\begin{array}{r}23.973 \\
(11.095)\end{array}$ & $\begin{array}{r}17.960 \\
(11.309)\end{array}$ & $\begin{array}{l}20.575 \\
(9.643)\end{array}$ & $\begin{array}{r}27.926 \\
(11.361)\end{array}$ \\
\hline NTRAB2 & $\begin{array}{r}669.790 \\
(572.983)\end{array}$ & $\begin{array}{r}697.804 \\
(575.211)\end{array}$ & $\begin{array}{r}450.325 \\
(504.283)\end{array}$ & $\begin{array}{r}516.341 \\
(460.130)\end{array}$ & $\begin{array}{r}908.959 \\
(621.858)\end{array}$ \\
\hline TPARO1 & $\begin{array}{r}0.251 \\
(0.434)\end{array}$ & $\begin{array}{r}0.257 \\
(0.437)\end{array}$ & $\begin{array}{r}0.207 \\
(0.406)\end{array}$ & $\begin{array}{r}0.258 \\
(0.437)\end{array}$ & $\begin{array}{r}0.256 \\
(0.436)\end{array}$ \\
\hline TPARO2 & $\begin{array}{r}0.483 \\
(0.499)\end{array}$ & $\begin{array}{r}0.473 \\
(0.499)\end{array}$ & $\begin{array}{r}0.561 \\
(0.496)\end{array}$ & $\begin{array}{r}0.489 \\
(0.499)\end{array}$ & $\begin{array}{r}0.455 \\
(0.498)\end{array}$ \\
\hline TPARO3 & $\begin{array}{r}0.264 \\
(0.441)\end{array}$ & $\begin{array}{r}0.268 \\
(0.443)\end{array}$ & $\begin{array}{r}0.230 \\
(0.421)\end{array}$ & $\begin{array}{r}0.252 \\
(0.434)\end{array}$ & $\begin{array}{r}0.287 \\
(0.452)\end{array}$ \\
\hline LPRELAT & $\begin{array}{r}0.364 \\
(0.382)\end{array}$ & $\begin{array}{r}0.366 \\
(0.384)\end{array}$ & $\begin{array}{r}0.341 \\
(0.368)\end{array}$ & $\begin{array}{r}0.360 \\
(0.376)\end{array}$ & $\begin{array}{r}0.374 \\
(0.393)\end{array}$ \\
\hline CCAA1 & $\begin{array}{r}0.345 \\
(0.475)\end{array}$ & $\begin{array}{r}0.335 \\
(0.472)\end{array}$ & $\begin{array}{r}0.427 \\
(0.495)\end{array}$ & $\begin{array}{r}0.363 \\
(0.481)\end{array}$ & $\begin{array}{r}0.302 \\
(0.459)\end{array}$ \\
\hline CCAA2 & $\begin{array}{r}0.327 \\
(0.469)\end{array}$ & $\begin{array}{r}0.330 \\
(0.470)\end{array}$ & $\begin{array}{r}0.304 \\
(0.460)\end{array}$ & $\begin{array}{r}0.317 \\
(0.465)\end{array}$ & $\begin{array}{r}0.346 \\
(0.475)\end{array}$ \\
\hline ССААЗ & $\begin{array}{r}0.326 \\
(0.468)\end{array}$ & $\begin{array}{r}0.334 \\
(0.471)\end{array}$ & $\begin{array}{r}0.267 \\
(0.442)\end{array}$ & $\begin{array}{r}0.319 \\
(0.466)\end{array}$ & $\begin{array}{r}0.350 \\
(0.477)\end{array}$ \\
\hline ANTIG1 & $\begin{array}{r}0.242 \\
(0.428)\end{array}$ & $\begin{array}{r}0.183 \\
(0.387)\end{array}$ & $\begin{array}{r}0.703 \\
(0.457)\end{array}$ & $\begin{array}{r}0.296 \\
(0.456)\end{array}$ & $\begin{array}{r}0.052 \\
(0.223)\end{array}$ \\
\hline ANTIG2 & $\begin{array}{r}0.239 \\
(0.427)\end{array}$ & $\begin{array}{r}0.248 \\
(0.432)\end{array}$ & $\begin{array}{r}0.175 \\
(0.380)\end{array}$ & $\begin{array}{r}0.371 \\
(0.483)\end{array}$ & $\begin{array}{r}0.104 \\
(0.306)\end{array}$ \\
\hline ANTIG3 & $\begin{array}{r}0.517 \\
(0.499) \\
\end{array}$ & $\begin{array}{r}0.567 \\
(0.495) \\
\end{array}$ & $\begin{array}{r}0.121 \\
(0.326) \\
\end{array}$ & $\begin{array}{r}0.331 \\
(0.470) \\
\end{array}$ & $\begin{array}{r}0.842 \\
(0.364) \\
\end{array}$ \\
\hline $\mathrm{N}^{\circ}$ Obs. & 6546 & 5805 & 741 & 3122 & 2683 \\
\hline
\end{tabular}

Nota: valores medios y entre paréntesis los errores estándar.

Fuente: Elaboración propia a partir de los datos procedentes de la Encuesta de Condiciones de Vida para el año 2010 (I.N.E.). 
